\title{
S-Adenosyl Methionine (SAMe) versus Escitalopram and Placebo in Major Depression RCT: Efficacy and Effects of Histamine and Carnitine as Moderators of Response
}

Jerome Sarris, PhD, MHSc,1,2

George I Papakostas, MD, $\mathrm{PhD}^{3}$

Ottavio Vitolo, $\mathrm{MD}^{3}$

Maurizio Fava, $\mathrm{MD}^{3}$

David Mischoulon, $\mathrm{MD}, \mathrm{PhD}^{3}$

${ }^{1}$ The University of Melbourne, Department of Psychiatry

2 Swinburne University of Technology, Centre for Human Psychopharmacology

3 Depression Clinical and Research Program, Department of Psychiatry, Massachusetts General Hospital, Harvard Medical School

Clinicaltrials.gov identifier: NCT00101452

Word Count: 3180

Figures and Tables: 3

\section{Conflicts of Interest:}

No direct conflicts of interest noted. See end of manuscript for general disclosures

\section{Correspondence:}

Dr Jerome Sarris

The Melbourne Clinic, Department of Psychiatry

The University of Melbourne

2 Salisbury St, Richmond, Melbourne, Australia

Ph:+61394209350 E: jsarris@unimelb.edu.au 


\begin{abstract}
Objective: To assess the antidepressant efficacy of S-adenosyl methionine (SAMe), a naturally occurring methyl donor, versus the selective serotonin reuptake inhibitor (SSRI) escitalopram and a placebo control; and to determine whether serum histamine or carnitine levels modified treatment response.

Methods: We examined a subsample $(n=144)$ from one site of a two-site study of adults with diagnosed Major Depressive Disorder (MDD), recruited from 4/13/05 to $12 / 22 / 09$, who consented to the additional blood draw for serum histamine and carnitine levels. After washout, eligible subjects were randomized to SAMe (1600mg$3200 \mathrm{mg} /$ daily), escitalopram (10mg-20mg/daily), or matching placebo for 12 weeks of double-blind treatment (titration at week 6 in non-response).

Results: On the primary outcome of the Hamilton Depression Rating Scale (HAMD-17), a significant difference in improvement was observed between groups from baseline to week 12 ( $p=0.039)$. The effect size from baseline to endpoint was moderate to large for SAMe versus placebo $(d=0.74)$. SAMe was superior to placebo from week 1 , and to escitolapram during weeks 2,4 , and 6 . No significant effect was found between escitalopram and placebo or SAMe. Response rates (HAMD-17 $>50 \%$ reduction) at endpoint were $45 \%, 31 \%$, and $26 \%$ for SAMe, escitalopram, and placebo, respectively; while remission rates (HAM-D $<7)$ were $34 \%$ for SAMe $(p=0.003), 23 \%$ for escitalopram $(p=0.023)$, and $6 \%$ for placebo. No correlation between baseline histamine level and reduction of HAMD-17 score was found for either the SAMe or escitalopram groups. Baseline carnitine levels were also not found to moderate response to either treatment.

Limitations: While SAMe appears to be an effective antidepressant agent, the overall findings from the parent study (which showed no significant difference between groups due to site differences) must be taken into consideration.

Conclusions: These preliminary results provide encouraging evidence for the use of SAMe in the treatment of MDD. Histamine and carnitine serum level may not necessarily moderate response to SAMe.
\end{abstract}

Key Words: S-Adenosyl Methionine, SAMe, Escitalopram, Antidepressant, Histamine, Carnitine, Depression, RCT, One-Carbon Cycle

Running Header: SAMe versus Escitalopram and Placebo in MDD 


\section{Introduction}

Major Depressive Disorder (MDD) is a common, typically recurrent and disabling disorder. Complementary and Alternative Medicine (CAM) use is prevalent by sufferers of mood disorders, with data from a nationally representative sample in the USA of 2,055 people interviewed during 1997-1998 revealing that 54\% of those with severe depression reported using CAM during the previous 12 months (Kessler, Soukup et al. 2001).

S-adenosyl methionine (SAMe) is a naturally occurring substance synthesized from the amino acid L-methionine and adenosine triphosphate through the one-carbon cycle, a metabolic pathway that relies in part on adequate levels of folate and B12 (Spillmann and Fava, 1996; Papakostas et al, 2009). Routinely prescribed in Europe for nearly 30 years, SAMe has gained popularity in the U.S. following its release as an over-the-counter dietary supplement in 1998-99 (Mischoulon and Fava, 2002). SAMe has been proposed as a potential treatment for a number of medical conditions, particularly MDD (Spillmann and Fava, 1996). Double-blind studies have demonstrated that parenteral or oral preparations of SAMe, compared with a number of standard tricyclic antidepressants (TCAs) such as clomipramine, amitriptyline, and imipramine, are generally equally effective, and tend to produce relatively fewer side effects (Bell et al, 1988; Delle Chiaie et al, 2002; Pancheri et al, 2002). Recent work from our group has supported efficacy of SAMe as an augmentation agent in partial and non-responders to SSRIs and SNRIs (Alpert et al, 2004; Papakostas et al, 2010)

One study thus far has compared the efficacy of SAMe versus both placebo and a standard selective serotonin reuptake inhibitor (SSRI) (Mischoulon et al, in press). These types of studies are important because, while SAMe may demonstrate equivalency with a standard antidepressant, an inactive comparator is necessary to rule out placebo effects. Likewise, if the natural product outperformed placebo, we need to know how this would compare to an active comparator. Finally, given the 
widespread use of SSRIs as first line antidepressants, many efficacy studies of novel putative antidepressants will use SSRIs as the standard comparator.

One proposed explanation for a number of psychiatric disorders that may align with the neurobiological action of SAMe is the histadelic model (Pfeiffer et al, 1970; Edelman, 2001). This model suggests that mood and thought disorders may be caused, at least in some individuals, by elevated levels of histamine in the brain. Some studies have suggested that depression, bipolar disorder, schizophrenia, and drug abuse are associated with abnormal histamine levels, in some instances in as many as $20 \%$ of cases (Pfeiffer, 1988). Elevated levels of histamine have been associated with severe depression, easy crying, insomnia, obsessive-compulsive ruminations, and suicidality (Pfeiffer, 1988). Under-methylation and low serotonin have been implicated among the mechanisms involved in the presenting mood and thought abnormalities observed in histadelia (Pfeiffer, 1988; Edelman 2001). Histadelic patients are not thought to respond well to conventional antidepressants or ECT. Investigation into the relationship between histadelia, depression, and response to treatment, could therefore prove valuable in the characterization and therapy of mood disorders.

Histamine is metabolized in part by SAMe, which methylates histamine's ring structure, thus forming N-methyl-histamine, a harmless metabolite (Pfeiffer, 1988; Edelman, 2001). Pfeiffer's model suggests that individuals with high levels of histamine would be especially likely to benefit from treatment with SAMe, and anecdotal data have supported this hypothesis. SAMe could potentially normalize histamine levels in the brain by methylating histamine. Conversely, depressed individuals with low or even normal histamine might not be expected to benefit as much from SAMe, assuming that histamine levels were playing a substantive role in their depression. Regardless, as SAMe modulates a range of neurochemical pathways, it still may be potentially effective in persons with low histamine. 
Histadelia may also be relevant to mood disorder mechanisms because elevated histamine also results in low serotonin (Edelman, 2001). It is therefore possible that administration of SSRI antidepressants may also have beneficial effects on histadelic individuals, and anecdotal evidence supports this idea. As it stands, there is limited systematic research on these issues, and data are limited to clinical anecdotes and studies with small subject samples.

Acetyl-L-carnitine (ALC) is an ester derivative of the trimethylated amino acid, Lcarnitine. ALC is synthesized in the human brain, liver, and kidney via the enzyme ALC-transferase. ALC is also available in the diet, in meat and dairy products. ALC facilitates uptake of acetyl CoA into the mitochondria during fatty acid oxidation, enhances acetylcholine production, and stimulates protein and membrane phospholipid synthesis (Indiveri et al, 2011). Several studies, including some doubleblind, placebo-controlled trials, have shown that ALC may be of benefit in treating geriatric depression (Tempesta et al, 1987), attention deficit disorder in children (Sarris et al, 2011), and cognitive impairment associated with dementia (Hudson and Tabet, 2003) or alcoholism (Tempesta et al, 1990). Despite a number of published investigations suggesting beneficial effects of ALC in the psychiatric population, there are no known studies measuring serum levels of carnitine in depressed individuals. While such a carnitine-related mechanism of depression would not seem to suggest a clear link to SAMe, if a carnitine deficiency plays a causative role in the development of depression, such an investigation would provide new insights into mechanisms of depression and approaches to treatment.

Due to these considerations, the primary aims of this research was to first assess the efficacy of SAMe versus escitalopram and placebo in treating MDD via 12 weeks of randomized, double-blind treatment (at the Massachusetts General Hospital site). The

results of the parent study (cf. Mischoulon et al. 2014) revealed no significant effects between groups across Time, however there was a significant effect in 


\section{favor of SAMe over placebo at weeks 8 and 10. A range of factors may account for this (highlighted in the Discussion below).}

Our second aim was to analyze the relationship between histamine and carnitine levels and their response; and third, to determine if histamine or carnitine levels changed during the course of antidepressant treatment. We hypothesized that subjects with higher baseline levels of serum histamine or lower levels of carnitine would be more likely to improve with SAMe treatment compared to those with lower levels of histamine and higher levels of carnitine. We also predicted that SAMe treatment would be associated with lowering of serum histamine levels.

\section{Methods}

Overview and Eligibility Requirements

The data were provided from a clinical trial site (Massachusetts General Hospital: MGH) of a two-site parent study involving the acute 12 -week controlled phase of a doubleblind, placebo-controlled administration of SAMe vs. escitalopram in 189 participants with MDD. This paper examines 144 subjects from the MGH site. The study was approved by the Partners Human Research Committee (IRB) and was registered on ClinicalTrials.Gov (NCT00101452).

The full methodology of the parent trial is detailed elsewhere (Mischoulon et al, in press). Consenting outpatients with MDD were recruited from 4/13/05 to 12/22/09. Inclusion criteria for eligibility into the study consisted of: participants with diagnosed MDD by the Structured Clinical Interview for DSM-IV Axis I Disorders - Patient Edition (SCID-I/P; First et al, 1995), aged 18-80 years old, and a score of 25 or greater on the Inventory of Depressive Symptomatology - Clinician-Rated (IDS-C) (Rush et al, 1996). Patients must have also had Clinical Global Impression Improvement (CGI) scores > 2 (i.e. less than much or very much improved) from the screen to the baseline visit. Exclusion criteria consisted of: pregnant women or women of child bearing potential 
who were not using a medically accepted means of contraception; patients with a range of psychiatric and medical disorders, or those on various medications; current use of other psychotropic drugs; previous moderate use of SAMe or escitolapram; ongoing psychotherapy; and multiple treatment failures.

\section{Study Procedures}

Following, if required, a two-week washout period for patients on antidepressants (fluoxetine required a four-week washout period), eligible and consenting subjects were randomly assigned to double-blind treatment with SAMe, escitalopram, or placebo for 12 weeks. Randomization was conducted via computerized random allocation by the research pharmacist, and the participants and assessors were both blinded to treatment. During the first week of the study and washout period, no medications were given. During the first six weeks of double-blind treatment, all patients were randomly assigned to one of three initial dosing regimens, with either SAMe tosylate disulfate $800 \mathrm{mg}$ twice per day $(1600 \mathrm{mg} /$ day divided into two doses, each dose containing 400mg of active SAMe ions: Group 1) or escitalopram (10 mg/day divided into two doses: Group 2); or double-dummy matching placebo (Group 3). A double-dummy design was employed whereby participants were given two bottles, with one bottle containing SAMe or SAMe placebo, and the other bottle containing escitalopram or escitalopram placebo. To maximize the probability of response, a dose increase was allowed for non-responders (patients with a $<50 \%$ HAM-D-17 reduction from baseline) at week 6. Dose decreases to the previous level were allowed in cases of intolerable side effects from titration.

The primary outcome measure used to assess depression, was the 17-item Hamilton-D Scale (HAMD-17) (Hamilton, 1960), while the Clinical Global Improvement-severity (CGI-S) (Guy, 1976) was used as a clinician-rated secondary outcome measure. Assessments were conducted at Baseline and at weeks 1,2,4,6,8,10, and12. SAMe and matching placebo were supplied by Pharmavite, CA. Escitalopram and matching placebo were purchased from Forest Pharmaceuticals. SAMe tosylate disulfate and 
SAMe-placebo tablets were prepared to be identical in appearance and texture to ensure double-dummy, blind conditions. Pill counts were carried out at every visit to determine compliance. Subjects were compensated $\$ 25$ for each completed visit.

In addition to normal testing procedures, study participants at MGH were asked to make two optional donations of $30 \mathrm{ml}$ of blood for the measurement of histamine and carnitine levels, collected at baseline and week 12 . Storing and analysis of the bloods was conducted at the Mayo Clinic Department of Medicine and Pathology, Rochester (histamine), and Quest Diagnostics, San Juan Capistrano (carnitine). For the histamine assay, plasma was separated within 30 minutes of collection, and samples were stored at - $20 \mathrm{C}$ until analyzed. The assay was a competitive displacement enzyme immunoassay from Immunotech, Marseilles, France. Bound enzymatic activity of histamine was measured by addition of a chromogenic substrate [para nitro phenyl phosphate]. Derivatized histamine competed with enzyme-labelled, acylated histamine for binding to a limited number of antibody sites on microtiter wells. For the carnitine assay, plasma was separated within 30 minutes of collection, and samples were stored at - $20 \mathrm{C}$ until analyzed. After addition of a mix of deuterated internal standards to the sample, proteins were precipitated and acylcarnitines were extracted by the addition of acetonitrile. The acylcarntine acid groups were derivatized by the addition of butanolic $\mathrm{HCl}$ to form butyl esters, and were analyzed by LC-ESI-MS/MS using a Thermo Finnegan TSQ Quantum instrument.

\section{Statistical Analysis}

Participants who met criteria for MDD and had a HAMD-17 score of $>14$ were analyzed as "intent-to-treat" using Last Observation Carried Forward (LOCF), in which all patients randomized to any of the three treatment arms had their data included in the analysis. The primary efficacy measure was the difference in change in HAMD-17 score across the three groups from baseline to week 12 
endpoint. A repeated measures ANCOVA, using baseline HAMD-17 score as the covariate (Van Breukelen 2006), was conducted to assess differences between Group $\mathrm{X}$ Time, while a one-way ANOVA was used to assess any differences between groups across each time period. Adequate response was calculated at week 4, 8, and 12, and was defined as a 50 percent or greater decrease in HAMD-17 score. Remission was defined as a HAMD-17 score of 7 or less at endpoint. Interactions for response and remission rates were assessed via Chi-Square test with a Bonferroni correction for multiple comparisons. A linear regression analysis (Spearman's rho, due to sample size) was utilized to examine whether histamine and carnitine levels correlated with change on the HAMD-17 score, while an ANOVA was used to determine any differences between SAMe and escitalopram baseline levels and week 12 levels. All tests were 2 -tailed, and significance (a) was set at 0.05 . We calculated the effect size $(d)$ by taking the between-group difference between means of the active and control groups at the start and end of the controlled phase, and dividing this by the pooled within-group standard deviation. Data were analyzed by SPSS 19.0.

Insert Figure 1 about here

\section{Results}

One hundred and 144 eligible participants (44\% female, mean age $47.6+13.1)$ with diagnosed MDD underwent the washout screening phase. Fifty four participants completed the study: SAMe $(n=18)$, escitalopram $(n=20)$, and placebo $(n=16)$, with no significant difference in attrition rates between groups. Upon study completion 102 participants meeting inclusion criteria had data available for analysis $\operatorname{SAMe}(n=32)$, escitalopram $(n=35)$, and placebo $(n=35)$. Seventy four percent $(74 \%)$ of the sample selfreported as Caucasian, 21\% Black, 4\% Hispanic, and 1\% Asian. Eighty-eight percent $(88 \%)$ had some college education or had graduated from college, 16\% had high school 
level education, while $12 \%$ did not graduate from high school. Forty three percent (43\%) of the sample had never been married, while $23 \%$ were currently in a relationship. Thirty-nine percent (39\%) were in some form of work, 26\% were unemployed, 14\% reported having a disability, 14\% were retired, and $7 \%$ were either students or volunteers. No significant differences were found between the groups on any baseline characteristic.

Baseline HAMD-17 scores were $19.09(+4.5), 20.83(+4.6)$, and $20.63(+4.4)$ for SAMe, escitalopram, and placebo, respectively, with no significant difference between groups $(p=0.24)$. At the conclusion of the study at week 12, all treatments resulted in a significant reduction of HAMD-17 scores over time $\left(\mathrm{F}_{1,100}=5.50, p=0.021\right)$. A mean (sd) reduction of 7.31 (5.96), 6.69 (5.70), and 4.00 (5.64) points on the HAMD-17 occurred for SAMe, escitolpram, and placebo, respectively. Repeated measures ANCOVA found a significant Group X Time interaction $\left(\mathrm{F}_{2,100}=3.36, p=0.039\right.$ : see Figure 1$)$. Significant Group X Time differences were found from week $2(p=0.002)$ through to endpoint week $12(p=0.021)$, with the greatest differences occurring from week 2 to week $6(p=0.002)$. A direct comparison between SAMe and placebo revealed a significant effect between baseline and endpoint $\left(F_{1,65}=5.89 ; p=0.018\right)$, with this effect occurring at every time point from week $1(p=0.04)$ to week 12 ( $p=0.007)$, i.e. separation of SAMe from placebo occurred within a week of treatment initiation and was maintained throughout the course of treatment. SAMe was also found to be superior to escitalopram during weeks 2,4 , and 6 , though this separation was lost from weeks 8-12 as the escitalopram arm improved further.

Direct comparison between escitalopram and placebo, however, did not reveal any significant Group X Time difference from baseline to endpoint $\left(F_{1,68}=2.44 ; p=0.12\right)$. Likewise, no significant differences were seen for escitalopram versus placebo at any time-point. The effect size from baseline to endpoint HAM-D reduction was moderate to large for SAMe versus placebo $(d=0.74)$, and moderate for escitalopram versus placebo $(d=0.60)$. 
Response rates (HAM-D $>50 \%$ reduction) for treatments over time are detailed in Figure 2. While no statistically significant differences were found on any time point, SAMe had a superior response rate over placebo over all time points and nearly reached significance at week $8\left(X^{2}{ }_{1,61}=3.39, p=0.066\right)$. At weeks 4,8 , and 12 , respective response rates were $32 \%, 48 \%$, and $45 \%$ for SAMe; $16 \%, 31 \%$, and $31 \%$ for escitalopram; and 16\%, 26\%, and 26\% for placebo. At week 12 endpoint, the remission rates $(\mathrm{HAM}-\mathrm{D}<7)$ were $34 \%$ for $\mathrm{SAMe}, 23 \%$ for escitalopram, and $6 \%$ for placebo. A significant difference was found between groups $\left(X^{2} 2,102=8.57 ; p=0.014\right)$. Individual comparisons in remission rates between placebo and treatments revealed a highly significantly difference for SAMe $(p=0.003)$, and for escitalopram $(p=0.023)$. On the secondary outcome, CGI-S was also significantly different between treatments and placebo from baseline to week 12 for $\operatorname{SAMe}(p=0.037)$, and for escitalopram $(p=0.026)$.

Blood assays of a sub-sample of 20 participants with a measurable level of histamine, found a mean average of $0.53 \mathrm{ng} / \mathrm{mL}(+0.34$ : reference range $0.5-1.4 \mathrm{nmol} / \mathrm{mL})$ for the SAMe group and $0.57 \mathrm{ng} / \mathrm{mL}(+0.43)$ for escitalopram. After SAMe treatment, histamine levels were found to be non-significantly $(p=0.21)$ reduced to $0.35 \mathrm{ng} / \mathrm{mL}(+0.36)$, while histamine slightly increased to $0.63 \mathrm{ng} / \mathrm{mL}(+0.51)$ in the escitalopram group. No correlation between baseline histamine level and reduction on the HAMD-17 was found for the SAMe group $(r=0.36 ; p=0.12)$, nor for escitalopram $(r=-0.23 ; p=0.37)$. Furthermore, no significant differences were found between people who had a reduction of histamine from baseline to week 12, with response or remission rates (not shown). Baseline carnitine levels for SAMe were $7.07 \mathrm{ng} / \mathrm{mL}(+2.80)$, and for escitalopram $6.89 \mathrm{ng} / \mathrm{mL}$ (+1.92.) (normal reference range 4.04-12.19 nmol/mL). Baseline levels were not found to have any relationship to response for SAMe $(r=-0.14 ; p=0.60)$ or escitalopram ( $r=0.08 ; p=0.78)$. Interventions were well tolerated with no significant adverse effects.

Insert Figures 2, 3 about here 


\section{Discussion}

Our results suggest that SAMe is significantly more effective than placebo in reducing depressed mood, while the SSRI comparator did not achieve this result. The effect size was moderate to large for SAMe indicating that a strong clinical effect occurred. This effect was maintained throughout the study, except that as the ANCOVA slope pattern depicts, the HAMD-17 scores rose slightly during the last two weeks for the SAMe group. In the escitalopram group, the ANCOVA slope revealed a steady reduction of depression, while as expected, the placebo group had a steady re-correction with a rise in depression from week 6.

The findings of this investigation need to be interpreted in the context of the parent clinical trial, which was essentially a failed study, in which all 3 treatment arms produced a significant clinical improvement, but neither active treatment separated from placebo. It should still be noted however that the MGH sample analyzed in this paper contained 144 out 189 participants (76.2\%) of the overall study sample. The more encouraging findings from the MGH-based sample suggest that a site-effect occurred, revealing a significant effect from treatment (SAMe). It is not entirely clear why this may have happened, given that both sites were managed by experienced clinicians, well versed in the procedures of clinical trials. Regardless, it is interesting to note that an analysis of the data for a Site $x$ Treatment effect revealed a trend for a significant interaction $(p=0.078)$. One explanation may involve a gender difference between sites. A further investigation revealed that there was a gender difference between the sites with the Butler Hospital site having a $69 \%$ female sample compared to the Massachusetts General Hospital site having a $41 \%$ female sample $\left(X^{2} p<0.0001\right)$. No other between site differences of participant characteristics was found (e.g. depression severity, comorbidities, duration of episode). 
Strengths of this study include the use of an RCT design with an established SSRI comparator, and a relatively long treatment period. Of interest was that while results showed histamine levels were reduced by SAMe administration, baseline serum levels did not moderate response. This association cannot be discounted, however, because the sample size was underpowered due to one third of the sample either having no measurable histamine level, or not consenting for the blood tests. The regression slope ( $r$ of 0.34 ) for histamine in the SAMe group suggests that there is potential for participants with higher baseline levels of histamine to have a poorer response to treatment, which is the opposite of what we expected. It is possible that higher histamine levels are not so tractable to antidepressants, thus remaining an obstacle to effective response. Baseline carnitine levels also did not appear to moderate response to either treatment, though as discussed above, it would be premature to rule out this relationship. For instance, plasma levels of carnitine may not be indicative of levels of carnitine in the brain. Future research should explore histadelic effects in a much larger sample.

Even if there is no substantive relationship between SAMe and regulation of histamine and carnitine levels, there are various other neurochemical effects that may account for the therapeutic effect of SAMe, including enhanced methylation of catecholamines and increased serotonin turnover (Agnoli et al, 1977; Bottiglieri et al, 1986), reuptake inhibition of norepinephrine (Cimino et al, 1984), enhanced dopaminergic activity (Agnoli et al, 1977; Carney et al, 1986), decreased prolactin secretion (Bottiglieri et al, 1986; Fava et al, 1990), increased conversion of phosphatidylethanolamine to phosphatidylcholine (Cimino et al, 1984), and a potential decrease in the microviscosity of platelet membranes (Cohen et al, 1987).

Limitations to this paper are recognized. First, because the sample size for the histamine and carnitine analyses was modest, we cannot confirm that these biomarkers are not salient to predicting response to treatment. Second, we may not be able to 
generalize these findings to other populations e.g. adolescent or geriatric. Third, because the data were analyzed from only one site of a two-site parent study, we cannot know what impact histamine and carnitine might have shown had the entire sample been analyzed for these biomarkers. Likewise, because the efficacy analysis of the MGH sample is post-hoc, its inconsistency with the findings of the sample as a whole needs to be interpreted with caution.

In respect to clinical considerations, prescription of SAMe can usually be provided in the form of $200 \mathrm{mg}-400 \mathrm{mg}$ SAMe tablets at a total dose of $1600-3200 \mathrm{mg}$ of SAMe per day (equivalent to $800 \mathrm{mg}-1600 \mathrm{mg}$ of active SAMe ions). SAMe is an unstable molecule, thus it is advised to only use enteric coated tablets stored in blister packs. Some products may also advise refrigeration. Cost is another issue, with adequate dosage of a high quality SAMe prescription costing potentially approximately US\$80 per week. Anecdotally, SAMe may also cause stimulation (and potential switching in bipolar disorder), so it may be advised to take it in the morning and/or after lunch in patients with sleep disturbance (although our data did not show any significant difference between treatments for this effect).

In conclusion, our findings from this one site analysis provide encouraging evidence that SAMe is as an effective antidepressant agent, at least equivalent to the SSRI comparator escitalopram, and superior to placebo. Regardless, these results must be taken in context with the overall findings from the parent study. Preliminary evidence indicates that baseline histamine or carnitine levels do not necessarily moderate the effect of response to either SAMe or placebo. Further investigation of the impact of these biomarkers on antidepressant treatment response is warranted. 


\section{References}

Agnoli A, Ruggieri S, Cerone G, Aloisi, P, Baldassarre, M, 1977. The dopamine hypothesis of depression: results of treatment with dopaminergic drugs, in Garattini S (ed): Depressive Disorders. Stuttgard, Schattauer, 447-458.

Alpert J.E, Papakostas G, Mischoulon D, Worthington J.J 3rd, Petersen T, Mahal Y, Burns A, Bottiglieri T, Nierenberg AA, Fava M., 2004. S-Adenosyl-Lmethionine (SAMe) as an adjunct for resistant major depressive disorder: an open trial following partial or nonresponse to selective serotonin reuptake inhibitors or venlafaxine. J Clin Psychopharmacol. 24(6), 661-4.

Bell K.M, Plon L, Bunney W.E, Jr., Potkin, S.G, 1988. S-adenosylmethionine treatment of depression: a controlled clinical trial. Am J Psychiatry 145, 1110-1114.

Bottiglieri T, Carney MWP, Edeh J: A biochemical study of depressed patients receiving S-adenosyl-1-methionine (SAM), in Borchardt RT, Creveling CR, Ueland PM (eds): Biological Methylation and Drug Design Clifton, NJ, Humana Press, pp 327-338, 1986.

Carney M.W, Edeh J, Bottiglieri T, Reynolds, E.M, Toone, B.K, 1986. Affective illness and s-adenosyl methionine: a preliminary report. Clin Neuropharmacol 9, 379-385.

Cimino M, Vantini G, Algeri S, Curatola, G, Pezzoli, C, Stramentinoli, G, 1984. Age-related modification of dopaminergic and beta-adrenergic receptor system: restoration to normal activity by modifying membrane fluidity with S-adenosylmethionine. Life Sciences 34, 2029-2039.

Cohen BM, Satlin A, Zubenko GS: S-adenosyl-1-methionine in the treatment of Alzheimer's Disease. J Clin Psychopharmacol 7, 254-257, 1987.

Della Chiaie R, Boissard G, 1997. Meta-analysis of 2 European multicenter controlled trials with ademethionine (SAMe) in major depression. Biol Psychiatry 42, suppl 1,245S.

Edelman E. Natural Healing for Schizophrenia: And Other Common Mental Disorders, 2001. Borage Books.

Fava M, Rosenbaum J.F, MacLaughlin R, Falk W.E, Pollack M.H, Cohen L.S, Jones L, Pill L, 1990. Neuroendocrine effects of s-adenosyl-1-methionine, a novel putative antidepressant. J Psychiatric Res 24,177-184.

First B.M, Spitzer RL, Gibbon M, Williams J.B.W, 1995. Structured Clinical Interview for DSM-IV Axis I Disorders - Patient Edition (SCID I/P). Biometrics Research Department, New York State Psychiatric Institute.

Hamilton M, 1960. A rating scale for depression. J Neurol Neurosurg Psychiatry 23, 56-62.

Hudson S, Tabet N. Acetyl-L-carnitine for dementia, 2003. Cochrane Database Syst Rev, (2):CD003158.

Guy W, Bonato R.E., 1970. CGI: Clinical Global Impressions. Manual for the ECDEU Assessment Battery 2 Rev ed Chevy Chase, Md: National Institute of Mental Health. 
Indiveri $\mathrm{C}$, Iacobazzi $\mathrm{V}$, Tonazzi $\mathrm{A}$, Giangregorio $\mathrm{N}$, Infantino $\mathrm{V}$, Convertini $\mathrm{P}$, Console L, Palmieri F, 2011. The mitochondrial carnitine/acylcarnitine carrier: Function, structure and physiopathology. Mol Aspects Med.

Kessler R.C, Soukup J, Davis R.B, Foster D.F, Wilkey S.A, et al., 2001. The use of complementary and alternative therapies to treat anxiety and depression in the United States. Am J Psychiatry 158, 289-294.

Mischoulon D, Fava M, 2002. Role of S-adenosyl-L-methionine in the treatment of depression: a review of the evidence. Am J Clin Nutr 76, 5, 1158S-61S.

Mischoulon D, Price L.H, Carpenter L.L, Tyrka A.R, Papakostas G.I, Baer L, Dording C.M, Clain A.J, Durham K, Walker R, Ludington E, Fava, M, 2014. A Double-Blind, Randomized, Placebo-Controlled Clinical Trial of SAdenosyl-L-Methionine (SAMe) Vs. Escitalopram in Major Depressive Disorder. J Clin Psychiatry (in press).

Pancheri P, Scapicchio P, Chiaie R.D: A double-blind, randomized parallel-group, efficacy and safety study of intramuscular S-adenosyl-L-methionine 1,4butanedisulphonate (SAMe) versus imipramine in patients with major depressive disorder, 2002. Int J Neuropsychopharmacol 4, 287-94.

Papakostas G.I, Mischoulon D, Shyu I, Alpert J.E, Fava M, 2010. S-adenosyl methionine (SAMe) augmentation of serotonin reuptake inhibitors for antidepressant nonresponders with major depressive disorder: a doubleblind, randomized clinical trial. Am J Psychiatry 167, 8, 942-8.

Papakostas G.I., 2009. Evidence for S-adenosyl-L-methionine (SAM-e) for the treatment of major depressive disorder. J Clin Psychiatry, 70 Suppl 5:18-22.

Pfeiffer CC, 1988. Nutrition and Mental Illness: An Orthomolecular Approach to Balancing Body Chemistry. Healing Art Press, 24-32.

Pfeiffer C.C, Iliev V, Goldstein L, Jenney E.H, Schultz R, 1970. Blood histamine, polyamines and the schizophrenias. Computer correlations of the low and high blood histamine types. Res Commun Chem Pathol Pharmacol 2,24765.

Rush AJ, Gullion CM, Basco MR, Jarrett RB, Trivedi MH: The Inventory of Depressive Symptomatology (IDS): psychometric properties. Psychol Med. 1996 May;26(3):477-86.

Sarris J, Kean J, Schweitzer I, Lake J, 2011. Complementary medicines (herbal and nutritional products) in the treatment of Attention Deficit Hyperactivity Disorder (ADHD): a systematic review of the evidence. Complement Ther Med 4, 216-27.

Spillmann M, Fava M, 1996. S-adenosyl-methionine (ademethionine) in psychiatric disorders. CNS Drugs 6:416-425.

Tempesta E, Casella L, Pirrongelli C, et al., 1987. L-acetylcarnitine in depressed elderly subjects. A cross-over study vs placebo. Drugs Exp Clin Res, 3,417423.

Tempesta E, Troncon R, Janiri L, Colusso L, Riscica P, Saraceni G, Gesmundo E, Calvani M, Benedetti N, Pola P, 1990. Role of acetyl-L-carnitine in the treatment of cognitive deficit in chronic alcoholism. Int $\mathrm{J}$ Clin Pharmacol Res $10(1-2), 101-7$. 
Van Breukelen, 2006. ANCOVA versus change from baseline: more power in randomiszed studies, more bias in nonrandomized studies. Journal of clinical epidemiology 9, 59. 
Figure 1. HAM-D-17 Score Reduction over 12 weeks of Treatment ( $n=144)$

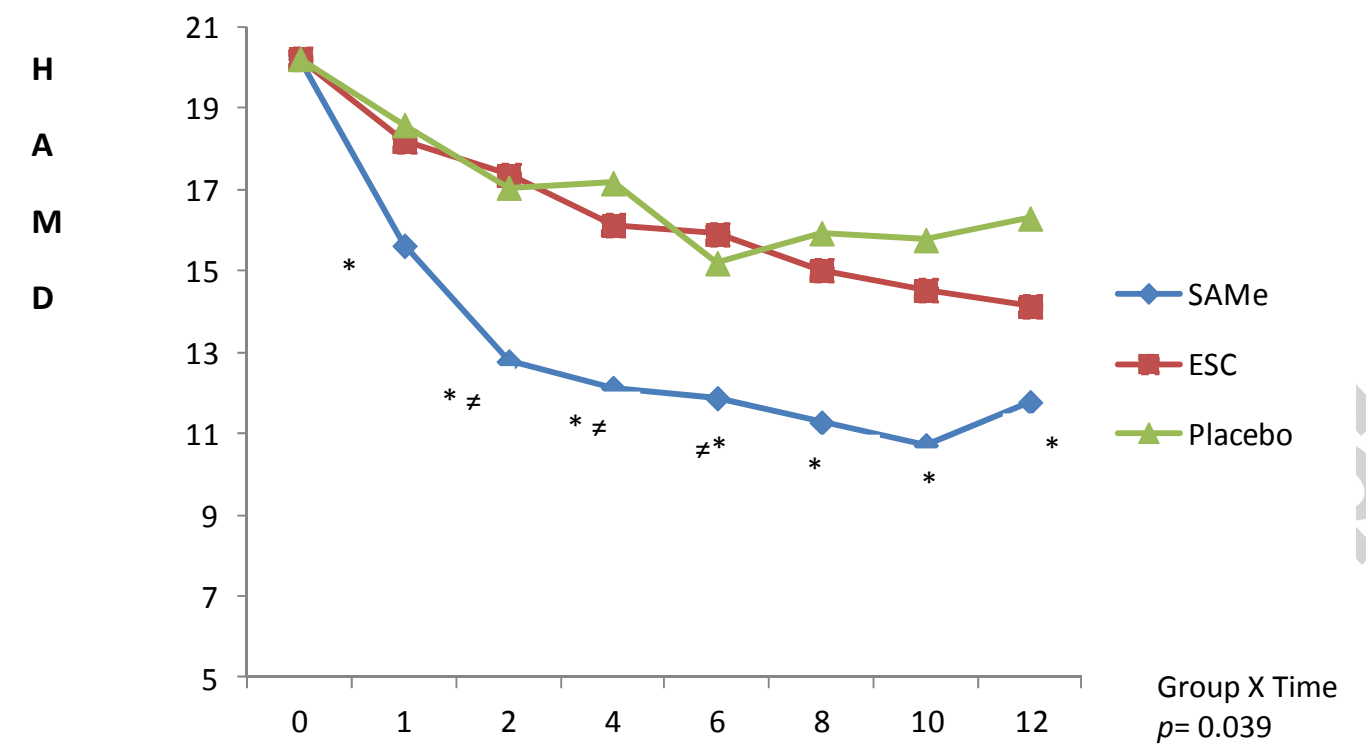

SAMe $n=32$; SSRI $n=35$; Placebo $n=35$; * SAMe $p<0.05$ vs. placebo; \# SAMe $p<0.05$ vs. ESC

$\mathrm{HAMD}=$ Hamilton Depression Rating Scale; $\mathrm{SAMe}=\mathrm{S}-$ Adenosyl Methionine, $\mathrm{ESC}=$ Escitalopram 
Figure 2. Response Rates on HAM-D-17* $(n=144)$

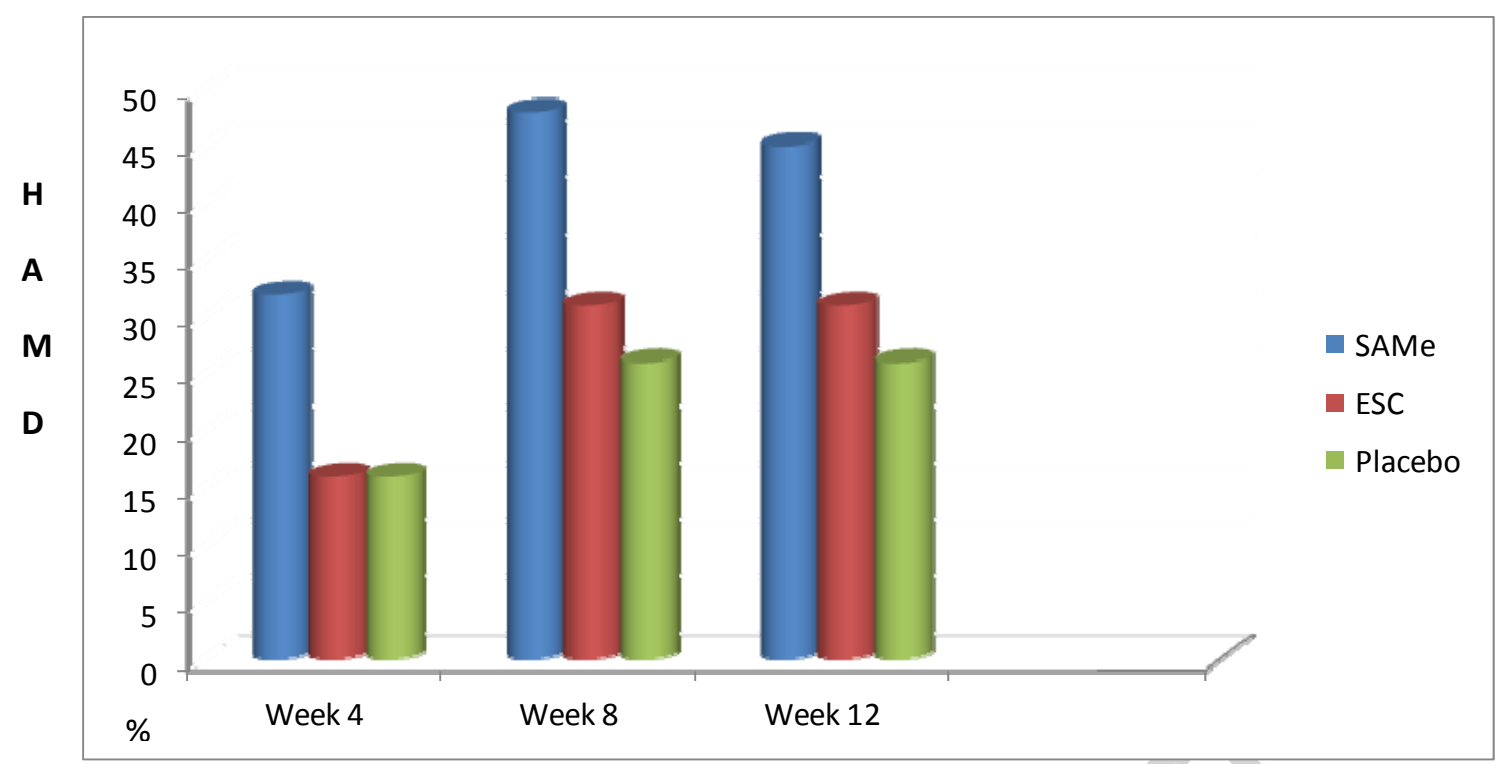

* Response rate $=$ Reduction of HAM-D-17score of $50 \%$ or greater from baseline $\mathrm{ESC}=$ Escitalopram

Figure 3. Remission Rates on HAM-D-17 at Week $12 *(n=144)$

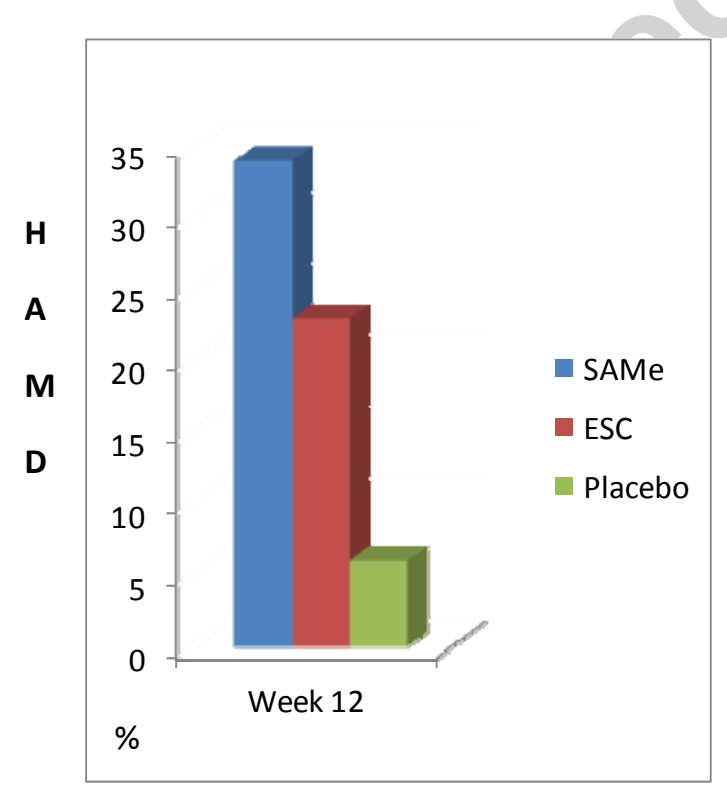

* Remission rate= HAM-D-17 of 7 or below at week 12. ESC $=$ Escitalopram 


\section{Funding Source}

The parent study was supported by grant R01AT001638-01A1 to Dr Maurizio Fava, from the National Center for Complementary and Alternative Medicine (NCCAM).

Dr Jerome Sarris was funded by an Australian National Health \& Medical Research Council fellowship (NHMRC funding ID 628875), in a strategic partnership with The University of Melbourne and The Centre for Human Psychopharmacology at Swinburne University of Technology.

Dr David Mischoulon was funded by a grant from the Bowman Family Foundation.

\section{Contributors}

Drs Sarris, Fava, Mischoulon, Papakostas, and Vitiolo have all read and approved this manuscript. All authors contributed significantly to the intellectual property, design, writing and proofing of the manuscript.

\section{Conflicts of Interest}

No direct conflicts of interest are noted by any author.

Dr Sarris is involved in academic research of natural products in the treatment of psychiatric disorders. This publication is not supported by any direct funding and no potential financial benefit is expected as a result of this publication. Dr Sarris has received honoraria, research support, royalties, or consultancy or travel grant funding from Integria Health, Blackmores, Bioceuticals, Taki Mai, Pepsico, HealthEd, Soho-Flordis, Pfizer, Elsevier, and the Society for Medicinal Plant and Natural Product Research.

Dr. Papakostas has served as a consultant for AstraZeneca, Bristol-Myers Squibb, Eli Lilly, GlaxoSmithKline, Evotec AG, Inflabloc Pharmaceuticals, Jazz Pharmaceuticals, Otsuka Pharmaceuticals, Pamlab, Pfizer, Pierre Fabre Laboratories, Shire Pharmaceuticals, and Wyeth; has received honoraria from 
AstraZeneca, Bristol-Myers Squibb, Eli Lilly, Evotec AG, GlaxoSmithKline, Inflabloc Pharmaceuticals, Jazz Pharmaceuticals, Lundbeck, Otsuka Pharmaceuticals, Pamlab, Pfizer, Pierre Fabre Laboratories, Shire Pharmaceuticals, Titan Pharmaceuticals, and Wyeth; he has received research support from Bristol-Myers Squibb, Forest Pharmaceuticals, the National Institute of Mental Health, Pamlab, Pfizer, and Ridge Diagnostics (formerly known as Precision Human Biolaboratories); and he has served on the speaker's bureau for Bristol-Myers Squibb and Pfizer.

Dr. Fava has received research support from: Alkermes, Inc.;AstraZeneca; BioResearch; BrainCells Inc.; Bristol-Myers Squibb; CeNeRx BioPharma; Clinical Trials Solutions, LLC; Clintara, LLC; Covance; Covidien; Eli Lilly and Company; EnVivo Pharmaceuticals, Inc.; Euthymics Bioscience, Inc.; Forest Pharmaceuticals, Inc.; Ganeden Biotech, Inc.; GlaxoSmithKline; Icon Clinical Research; i3 Innovus/Ingenix; Johnson \& Johnson Pharmaceutical Research \& Development; National Alliance for Research on Schizophrenia \& Depression (NARSAD); National Center for Complementary and Alternative Medicine (NCCAM); National Institute of Drug Abuse (NIDA); National Institute of Mental Health (NIMH); Novartis AG; PamLab, LLC.; Pfizer Inc.; Pharmavite ${ }^{\circledR}$ LLC; Photothera; Roche Pharmaceuticals; RCT Logic, LLC; Sanofi-Aventis US LLC; Synthelabo; Wyeth-Ayerst Laboratories. He has served as Advisor/Consultant to: Abbott Laboratories; Affectis Pharmaceuticals AG; Alkermes, Inc.; Amarin Pharma Inc.; Aspect Medical Systems; AstraZeneca; Auspex Pharmaceuticals; Bayer AG; Best Practice Project Management, Inc.; BioMarin Pharmaceuticals, Inc.; Biovail Corporation; BrainCells Inc; Bristol-Myers Squibb; CeNeRx BioPharma; Cephalon, Inc.; Clinical Trials Solutions, LLC; CNS Response, Inc.; Compellis Pharmaceuticals; Cypress Pharmaceutical, Inc.; DiagnoSearch Life Sciences (P) Ltd.; Dinippon Sumitomo Pharma Co. Inc.; Dov Pharmaceuticals, Inc.; Edgemont Pharmaceuticals, Inc.; Eisai Inc.; Eli Lilly and Company; ePharmaSolutions; EPIX Pharmaceuticals, Inc.; Euthymics Bioscience, Inc.; Fabre-Kramer Pharmaceuticals, Inc.; Forest Pharmaceuticals, Inc.; GenOmind, LLC; GlaxoSmithKline; Grunenthal GmbH; i3 Innovus/Ingenis; Janssen Pharmaceutica; Jazz Pharmaceuticals, Inc.; Johnson \& Johnson Pharmaceutical Research \& Development, LLC; Knoll Pharmaceuticals Corp.; Labopharm Inc.; Lorex Pharmaceuticals; Lundbeck Inc.; MedAvante, Inc.; 
Merck \& Co., Inc.; MSI Methylation Sciences, Inc.; Naurex, Inc.; Neuronetics, Inc.; NextWave Pharmaceuticals; Novartis AG; Nutrition 21; Orexigen Therapeutics, Inc.; Organon Pharmaceuticals; Otsuka Pharmaceuticals; PamLab, LLC.; Pfizer Inc.; PharmaStar; Pharmavite ${ }^{\circledR}$ LLC.; PharmoRx Therapeutics; Precision Human Biolaboratory; Prexa Pharmaceuticals, Inc.; Puretech Ventures; PsychoGenics; Psylin Neurosciences, Inc.; Rexahn Pharmaceuticals, Inc.; Ridge Diagnostics, Inc.; Roche; RCT Logic, LLC; SanofiAventis US LLC.; Sepracor Inc.; Servier Laboratories; Schering-Plough Corporation; Solvay Pharmaceuticals, Inc.; Somaxon Pharmaceuticals, Inc.; Somerset Pharmaceuticals, Inc.; Sunovion Pharmaceuticals; Supernus Pharmaceuticals, Inc.; Synthelabo; Takeda Pharmaceutical Company Limited; Tal Medical, Inc.; Tetragenex Pharmaceuticals, Inc.; TransForm Pharmaceuticals, Inc.; Transcept Pharmaceuticals, Inc.; Vanda Pharmaceuticals, Inc.; Wyeth-Ayerst Laboratories He has received speaking and publishing honoraria from: Adamed, Co; Advanced Meeting Partners; American Psychiatric Association; American Society of Clinical Psychopharmacology; AstraZeneca; Belvoir Media Group; Boehringer Ingelheim GmbH; Bristol-Myers Squibb; Cephalon, Inc.; CME Institute/Physicians Postgraduate Press, Inc.; Eli Lilly and Company; Forest Pharmaceuticals, Inc.; GlaxoSmithKline; Imedex, LLC; MGH Psychiatry Academy/Primedia; MGH Psychiatry Academy/Reed Elsevier; Novartis AG; Organon Pharmaceuticals; Pfizer Inc.; PharmaStar; United BioSource,Corp.; Wyeth-Ayerst Laboratories Equity Holdings: Compellis.

Royalty/patent, other income: Patent for Sequential Parallel Comparison Design (SPCD) and patent application for a combination of azapirones and bupropion in Major Depressive Disorder (MDD), copyright royalties for the MGH Cognitive \& Physical Functioning Questionnaire (CPFQ), Sexual Functioning Inventory (SFI), Antidepressant Treatment Response Questionnaire (ATRQ), Discontinuation-Emergent Signs \& Symptoms (DESS), and SAFER. Patent for research and licensing of SPCD with RCT Logic; Lippincott, Williams \& Wilkins; World Scientific Publishing Co. Pte.Ltd

Dr Mischoulon has received research support from the Bowman Family Foundation, Bristol-Myers Squibb Co., Cederroth, FisherWallace, Ganeden, Lichtwer Pharma, Nordic Naturals, Laxdale (Amarin), Methylation Sciences, 
Inc. (MSI), and SwissMedica. He has received honoraria for consulting, speaking, and writing from Pamlab, Bristol-Myers Squibb Co., Nordic Naturals, Virbac, Pfizer, Reed Medical Education, and the Massachusetts General Hospital Psychiatry Academy. He has received royalties from Back Bay Scientific for PMS Escape, and from Lippincott Williams \& Wilkins for published book "Natural Medications for Psychiatric Disorders: Considering the Alternatives."

\section{Acknowledgments}

Dr Jerome Sarris is funded by an Australian National Health \& Medical Research Council fellowship (NHMRC funding ID 628875), in a strategic partnership with The University of Melbourne and the Brain Sciences Institute at Swinburne University of Technology 


\section{University Library}

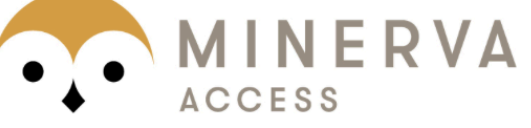

A gateway to Melbourne's research publications

Minerva Access is the Institutional Repository of The University of Melbourne

Author/s:

Sarris, J;Papakostas, GI;Vitolo, O;Fava, M;Mischoulon, D

Title:

S-adenosyl methionine (SAMe) versus escitalopram and placebo in major depression RCT: Efficacy and effects of histamine and carnitine as moderators of response

Date:

2014-08-01

Citation:

Sarris, J., Papakostas, G. I., Vitolo, O., Fava, M. \& Mischoulon, D. (2014). S-adenosyl methionine (SAMe) versus escitalopram and placebo in major depression RCT: Efficacy and effects of histamine and carnitine as moderators of response. JOURNAL OF AFFECTIVE DISORDERS, 164, pp.76-81. https://doi.org/10.1016/j.jad.2014.03.041.

Publication Status:

Accepted manuscript

Persistent Link:

http://hdl.handle.net/11343/41920 